\title{
Mitophagy Improves Ethanol Tolerance in Yeast: Regulation by Mitochondrial Reactive Oxygen Species in Saccharomyces cerevisiae
}

\author{
Hongjuan Jing*, Huanhuan Liu, Zhang Lu, Cui liuqing, and Xiaorong Tan* \\ College of Biological Engineering, Henan University of Technology, Zhengzhou 450001, P.R. China
}

\begin{abstract}
Ethanol often accumulates during the process of wine fermentation, and mitophagy has critical role in ethanol output. However, the relationship between mitophagy and ethanol stress is still unclear. In this study, the expression of ATG11 and ATG32 genes exposed to ethanol stress was accessed by real-time quantitative reverse transcription polymerase chain reaction (qRT-PCR). The result indicated that ethanol stress induced expression of the ATG11 and ATG32 genes. The colony sizes and the alcohol yield of atg 11 and atg 32 were also smaller and lower than those of wild type strain under ethanol whereas the mortality of mutants is higher. Furthermore, compared with wild type, the membrane integrity and the mitochondrial membrane potential of atg11 and atg32 exhibited greater damage following ethanol stress. In addition, a greater proportion of mutant cells were arrested at the $\mathrm{G1} / \mathrm{GO}$ cell cycle. There was more aggregation of peroxide hydrogen $\left(\mathrm{H}_{2} \mathrm{O}_{2}\right)$ and superoxide anion $\left(\mathrm{O}_{2}{ }^{-}\right)$in mutants. These changes in $\mathrm{H}_{2} \mathrm{O}_{2}$ and $\mathrm{O}_{2}{ }^{--}$in yeasts were altered by reductants or inhibitors of scavenging enzyme by means of regulating the expression of ATG11 and ATG32 genes. Inhibitors of the mitochondrial electron transport chain (mtETC) also increased production of $\mathrm{H}_{2} \mathrm{O}_{2}$ and $\mathrm{O}_{2}{ }^{--}$by enhancing expression of the $A T G 11$ and $A T G 32$ genes. Further results showed that activator or inhibitor of autophagy also activated or inhibited mitophagy by altering production of $\mathrm{H}_{2} \mathrm{O}_{2}$ and $\mathrm{O}_{2} \cdot$. Therefore, ethanol stress induces mitophagy which improves yeast the tolerance to ethanol and the level of mitophagy during ethanol stress is regulated by ROS derived from mtETC.
\end{abstract}

Keywords: Ethanol stress, mitophagy, reactive oxygen species, hydrogen peroxide, superoxide anion

Received: June 19, 2020 Accepted: September 22, 2020 First published online: September 25, 2020 *Corresponding authors H.Jing Phone: +86-371-67756513 E-mail: hjjing@haut.edu.cn X.Tan

Phone: +86-371-67756513 E-mail: tanxr2012@gmail.com

Supplementary data for this paper are available on-line only at http://jmb.or.kr.

pISSN 1017-7825 eISSN 1738-8872

Copyright(C) 2020 by The Korean Society for Microbiology and Biotechnology

\section{Introduction}

In eukaryotes, mitochondria are the main site of reactive oxygen species (ROSs) production. For example, superoxide anion $\left(\mathrm{O}_{2}{ }^{-}\right)$is produced by complex I and complex III of the electron transport chain in mitochondria (mtETC) $[1,2]$. Subsequently, $\mathrm{O}_{2}{ }^{-}$is immediately converted into less-reactive hydrogen peroxide $\left(\mathrm{H}_{2} \mathrm{O}_{2}\right)$ by mitochondrial manganese superoxide dismutase (Mn-SOD) [3-5]. Catalase (CAT) in mitochondria then converts $\mathrm{H}_{2} \mathrm{O}_{2}$ into water [6-8]. Therefore, the inhibition of Mn-SOD and CAT leads to increase of ROS in both cytoplasm and mitochondria. Mitochondria are initially injured by ROS, then the injured mitochondria produce more ROS, initiating a progressive cycle of damage. Therefore, preventing mitochondrial dysfunction is especially important for the maintenance of cellular homeostasis $[9,10]$. Several studies suggest that mitophagy has a vital role in mitochondrial quality control by eliminating redundant, injured and disfunctional mitochondria [9-13].

Mitophagy is a form of selective macroautophagy (hereafter referred to as autophagy) characterized by specific degradation mitochondria. A series of autophagy related genes (ATG)s have been identified in the budding yeast Saccharomyces cerevisiae $[14,15]$. Besides common autophagy related proteins, the completion of mitophagy requires additional specific proteins. A mitochondrial outer membrane protein ATG32, which function as a receptor for mitophagy, plays a key role in the regulation of mitophagy. The protein ATG32 recruits ATG8 and ATG11 to the surface of mitochondria to form a mitophagy initiation complex $[10,12,16]$. The inhibition expression of ATG32 leads to the suppression of mitophagy, and vice versa. The mutant atg 32 inhibits mitophagy, but has no effect on common autophagy [10]. In contrast, a key determinant for mitophagy efficiency is the induction of expression of the ATG32 gene [17]. Yeast ATG11, as the structural scaffold, participates in mitophagy by mean of interaction with receptor ATG32 $[10,12,18,19]$. Except that mitophagy, ATG11 also participates in other forms of selective autophagy [19], such as pexophage [20], ER-phagy [21], genotoxin-induced targeted autophagy (Eapena et al., 2017) [22], and ribophagy [23].

There is evidence that ROS production is elevated during the fermentation process [24], and that the production of ROS might also be a component of the yeast cellular response to a variety of fermentation stressors 
$[25,26]$. Zyrina et al. has reported that $\mathrm{H}_{2} \mathrm{O}_{2}$ in the mitochondrial matrix has a signaling role in ethanol tolerance by Yap1p [5]. Additional studies indicate that mild ROS, as a signal molecule, regulates mitophagy [10-12, 27].

With regards to improving ethanol output, it has been reported that $S$. cerevisiae disrupts mitophagy function [28]. Our group has proved that ethanol induces autophagy [29]. However, there are no published studies on the relationship between mitophagy and ethanol. And that function and mechanism of mitophagy in yeast remain unclear. In the current study, the effect of ethanol on the expression of ATG32 and ATG11 genes was firstly inquired. Then the differences in the cell viability, cell cycle and ROS content between wild type and atg 32 and atg11 mutants were assessed. The type and source of ROS in yeast were also further inquired in the study.

\section{Materials and Methods}

\section{Strain and Maintenance Medium}

The yeast strain used in this study was BY4742 which was supplied by Pro. Zhiwei Huang of East China University. The mutants of atg11 and atg32 were purchased from the company (Invitrogen, USA). The mutant atg11 was constructed by hygromycin stripe homologous replacement the gene of ATG11 and the same was the mutant atg 32. Then the cells of mutants were grown on yeast peptone dextrose (YPD) agar medium with $200 \mu \mathrm{g} /$ $\mathrm{ml}$ geneticin G418. YPD agar medium contained $2 \%$ glucose, $1 \%$ peptone, $5 \%$ yeast extract and $2 \%$ agar. Yeast cells of wild type could not grow on YPD medium contained G418 whereas cells of mutants could survival from the medium with G418. Fresh cells grown on YPD medium slants for $24 \mathrm{~h}$ were used in all experiments.

\section{Fermentation Conditions}

The yeast cells for all experiments, as starter cultures, were grown for $12 \mathrm{~h}$ in $100 \mathrm{ml}$ of YPD liquid medium at $30^{\circ} \mathrm{C}$ with $180 \mathrm{rpm}$. The yeast cells, as the experimental cultures, were inoculated with $5 \times 10^{5} \mathrm{CFU} \mathrm{ml}^{-1}$ of starter culture solution. According to the methodology [30], the fermentation of cells was grown in YPD liquid medium at $30^{\circ} \mathrm{C}$ with $180 \mathrm{rpm} .10 \%$ ethanol (v/v) by adding absolute ethyl alcohol was used as the ethanol stress. $100 \mu \mathrm{M} 2$ methoxyestradiol (2-ME) and 3-amino-1,2,4-triazole (3-AT), $5 \mu \mathrm{M}$ Rapamycin (Rapa), $10 \mu \mathrm{M}$ methyladenine (3MA), $2.5 \mathrm{mM}$ glutathione (GSH), $1 \mathrm{mM} \mathrm{N}$-acetyl-cysteine (NAC), $50 \mu \mathrm{M}$ antimycin A (AntiA) and rotenone (Rote) were applied to treat yeast cells, respectively. The yeast cells as samples were collected at 1,2, and $24 \mathrm{~h}$ for assessing fermentation and growth parameters. The alcohol content in fermentation medium was measured by method of densitometer.

\section{Assessment of Mortality and Plasma Membrane Integrity}

The mortality of cell and the plasma membrane integrity were both assessed by the fluorescent dye propidium iodide (PI) (Molecular Probes, Sigma, USA) vital staining with minor adaptations [31,32]. PI is a nuclear staining reagent and it is an analog of ethidium bromide that releases red fluorescence upon insertion of double-stranded DNA. PI can not pass through the cell membrane of living cells whereas can traverse the broken membrane of the death cell. Briefly, yeast cells $\left(10^{6}\right.$ cells $\left.\mathrm{ml}^{-1}\right)$ were stained by $20 \mu \mathrm{M}$ PI for $10 \mathrm{~min}$ at $37^{\circ} \mathrm{C}$. The mortality of cells was measured by flow cytometry. The plasma membrane integrity of cells was observed by fluorescence microscope. The fluorescence intensity were measured by fluorescence microplate with $540 \mathrm{~nm}$ excitation wavelength and $590 \mathrm{~nm}$ emission wavelength.

\section{Assessment of Mitochondrial Membrane Potential}

Based on described elsewhere [33], the mitochondrial membrane potential of cells was detected by rhodamine 123 (Rh123). Rh123, as a cationic dye, can transfer into the mitochondrial matrix with help of mitochondrial membrane potential. So, the fluorescence of the yeast cells was weak under normal circumstances. On the contrary, fluorescence of Rh123 in broken yeast was high because Rh123 was released from mitochondria by disruption of mitochondrial membrane potential.

\section{Cell Cycle Analysis}

According to a previously described procedure, cell cycle analysis was also performed by PI [31]. The yeast cells were harvested, washed, and fixed with ethanol $(70 \%$, vol $/ \mathrm{vol})$ for $30 \mathrm{~min}$ at $4^{\circ} \mathrm{C}$. Then, the cells were destructed cell wall by sonication and followed by treatment with RNase for $2 \mathrm{~h}$ at $37^{\circ} \mathrm{C}$ in $50 \mathrm{mM}$ sodium citrate buffer ( $\mathrm{pH} 7.5$ ). Subsequently, the cells were incubated with proteinase $\mathrm{K}\left(1 \mathrm{mg}\right.$ per $10^{6}$ cells). Cell DNA was then stained overnight with PI (Molecular Probes, Sigma) at $4^{\circ} \mathrm{C}$. Flow cytometry was used to determined cells in each phase of the cell cycle.

\section{Assesement of Intercellular Reactive Oxygen Species \\ $\mathrm{H}_{2} \mathrm{O}_{2}$ was monitored by 2,7-dichlorodihydrofluorescein diacetate (DCFH-DA) (Molecular Probes, Sigma) essentially as described elsewhere [34]. DCFH-DA does not fluoresce and can traverse the cell membrane freely. DCFH-DA can be hydrolyzed by esterase to 2, 7-dichlorofluorescin (DCFH) which is arrested in an actively respiring cell. DCFH was oxidized by $\mathrm{H}_{2} \mathrm{O}_{2}$ to a fluorescent compound DCF. Dihydroethidium (DHE) (Molecular Probes, Sigma) as a probe was used to detect $\mathrm{O}_{2}{ }^{-}$. The yeast cells $\left(10^{6}\right.$ cells $\left.\mathrm{ml}^{-1}\right)$ were stained by $10 \mu \mathrm{M}$ DCFH-DA or $4 \mu \mathrm{M}$ DHE for $10 \mathrm{~min}$ at $30^{\circ} \mathrm{C}$ in dark. The fluorescences of DCF and DHE were detected by fluorescence microscope and flow cytometry.}

\section{Flow Cytometry Analysis}

Calibur flow cytometer (Becton, Dickinson and Company, USA) was used to detected fluorescence-activated cell sorter (FACS). The flow cytometer equipped with an argon ion laser emitting a $488 \mathrm{~nm}$ beam at $15 \mathrm{~mW}$. The 
green fluorescence was detected by a $550 \mathrm{~nm} /$ long-pass dichroic mirror with a $525 \mathrm{~nm} /$ band-pass filter. The red fluorescence was monitored by a $590 \mathrm{~nm} / \mathrm{long}$-pass with a $620 \mathrm{~nm} /$ band-pass filter. The green and red fluorescences were both collected by means of a $488 \mathrm{~nm}$ blocking filter. The green fluorescence (FL1 log) and the red fluorescence (FL2 log) were defined to measure by an acquisition protocol on a 4-decade logarithmic scale. The software for the Flow Jo System II acquisition and software cell quest pro were used to analyze data (20,000 cells per sample).

\section{qRT-PCR}

The RNA was extracted from the yeast cells of wild type by means of Trizol kit (Invitrogen). The destruction of cells was used by heat shock treatment with $15 \mathrm{~min}$ at $42^{\circ} \mathrm{C}$ followed by $3 \mathrm{~min}$ at $95^{\circ} \mathrm{C}$. Then, the SuperScript III Platinum Two-Step qRT-PCR kit with SYBR green (Invitrogen) was used to reverse transcribed the total RNA. The reverse-transcribed RNA was used as a template to amplify the genes, using primers to the ATG11 gene (sense, 5-TACAATCGTCTCCCTCGGTG-3; antisense, 5-TCCAAAGTGACAATTCTGCCTA-3), the ATG32 gene (sense, 5-AAATGTCGTTTCACCGTCTCA-3; antisense, 5-ACCATCATCATCTTGCTCGTTA-3) and to the ACT1 gene (sense, 5-GGATTCTGAGGTTGCTGCTTT-3; antisense, 5-TGACCCATACCGACCATGATAC-3). Real-time quantitative transcription polymerase chain reaction (qRT-PCR) was analyzed the expression of the expression of ATG11 and ATG32 genes by ABI Stepone plus (ABI). These results were normalized to the reference gene ACT1. Livak method or the $2^{-\Delta \Delta C T}$ method was used to analyze the data [35]. The equation was calculated as follows: $\Delta \Delta \mathrm{C}_{\mathrm{T}}=\left(\mathrm{C}_{\mathrm{T}}\right.$ (target gene $)-\mathrm{C}_{\mathrm{T}}($ reference gene $\left.)\right)$ test $-\left(\mathrm{C}_{\mathrm{T}}\right.$ (target gene $)-\mathrm{C}_{\mathrm{T}}($ reference gene $\left.)\right)$ calibrator. $\mathrm{C}_{\mathrm{T}}$ was referred to threshold cycle.

\section{Statistical Analysis}

Data were presented as means \pm standard deviations (SD) and the means were from at least three independent assays. To analysis the differential significance of the data, Student's $t$ test were carried out by Statistical Product and Service Solutions 19.0 (SPSS). Statistically significant or very significant were represented by $p$ values of less than 0.05 or 0.01 , respectively and were added a single or double asterisk at the tops of the columns in the figures.

\section{Results and Discussion}

\section{Ethanol Stress Induces Mitophagy}

It is established that ethanol accumulates gradually in S. cerevisiae during the fermentation process [24]. In order to confirm whether mitophagy is induced by ethanol stress, the expression of ATG11 and ATG32 genes under ethanol stress was measured by qRT-PCR. Results indicated that the expression of ATG11 and ATG32 genes was substantially increased by ethanol stress $(p<0.01)$ (Fig. 1A). The expression of ATG11 and ATG32 in yeast cells was increased to $278.3 \%$ and $194.0 \%$ by ethanol stress, respectively. Therefore, ethanol stress induces mitophagy and other selective forms of autophagy.

S. cerevisiae is a traditional fermentation microbe and has fewer mitochondria used for degradation compared to other yeast cells. It is difficult to induce mitophagy in a fermentable medium. Therefore, mitophagy is induced when cells are exposed to rapamycin (the Tor inhibitor) or nitrogen deficiency after pre-culturing in a fermentation medium [10]. In the current study, ethanol increased the expression of ATG11 and ATG32 genes within $4 \mathrm{~h}$ (Fig. 1). Moreover, it is well known that ATG11, as a scaffold molecule, particpates in many forms of selective autophagy [19-23]. Therefore, there is evidence that ethanol induces mitophagy as well as other forms selective autophagy.

\section{Mitophagy Increases Alcohol Output and Decreases Mortality in Yeasts Exposed to Ethanol Stress}

Although ATG11 contribute to many selective autophagy [20-23], the mutant atg11 is known to inhibit mitophagy [36]. In order to investigate the function of mitophagy during ethanol stress, the alcohol production of wild type and mitophagy mutants atg11 and atg32 was assessed in normal YPD medium. Results indicated that although the growth curves of the wild type and mutants were not different in the absence of ethanol stress (Fig. S1) the alcohol output of $\operatorname{atg} 11$ and $\operatorname{atg} 32$ was significantly lower than that of the wild type (Fig. 1B). In contrast, the colony sizes of all strains were smaller under ethanol stress (Fig. 1C), and the colony sizes of the mutants were much smaller compared to wild type colonies (Fig. 1C). In addition, ethanol stress increased the cell death in all strains (Fig. 1D) and the mortality of atg11 and atg32 was remarkably higher than that of wild type under ethanol stress (Fig. 1D). For example, the mortality of wild type, atg11 and atg 32 with ethanol for $1 \mathrm{~h}$ was $10.6 \%, 17.8 \%$, and $15.8 \%$, respectively. These results suggest that mitophagy increases alcohol production by protecting yeast injure from ethanol stress.

The accumulation of ethanol in a medium inhibits the growth and viability of yeast cells [30,37,38]. And a similar finding was made in the present study. Ethanol decreased the growth rate and promoted cell death in all strains (Figs. 1C and 1D). In addition, it has been reported that the biomass yield of the atg 32 is decreased, whereas the output of ethanol is increased [28]. Similarly, atg32 and atg 11 were decreased the growth rate and were increased the death rate by ethanol stress (Figs. 1C and 1D). In contrast, alcohol output of mitophagy mutants was decreased (Fig. 1B). It is suggested that the death of yeasts by ethanol stress occurs during the early fermentation stage. Alcohol production requires the living yeast to transfer carbohydrate to alcohol throughout the entire fermentation process. For example, reducing agents increased the alcohol output (Fig. S4) through an attenuation of the cell death (Fig. S2A). In contrast, antioxidase inhibitors reduced alcohol yield (Fig. S4) by increasing the cell death (Fig. S2B).

Mitophagy Contributes to the Maintenance of Mitochondrial Membrane Potential and Cell Cycle of Yeast Cells

Ethanol gradually accumulates in the culture broth during the process of fermentation. S. cerevisiae, an efficient ethanol producer, has a high tolerance to ethanol [38-40]. However, high levels of ethanol eventually disrupts cell 
A

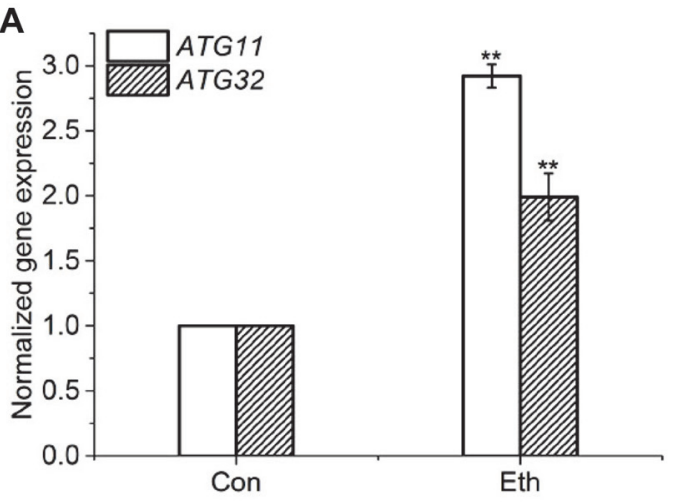

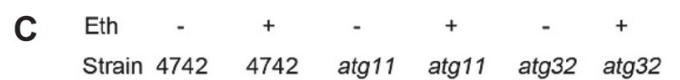

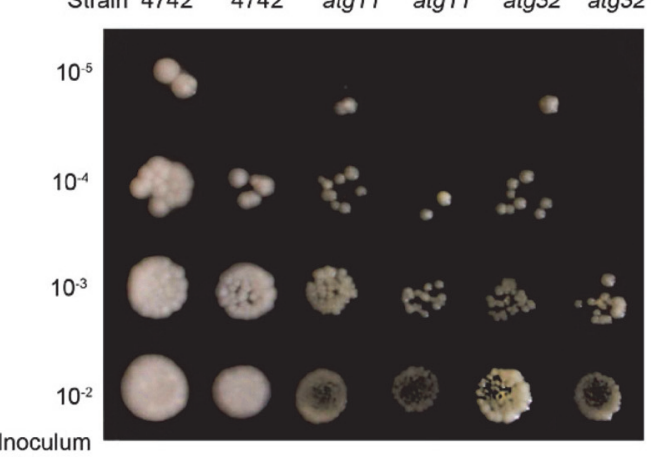

B

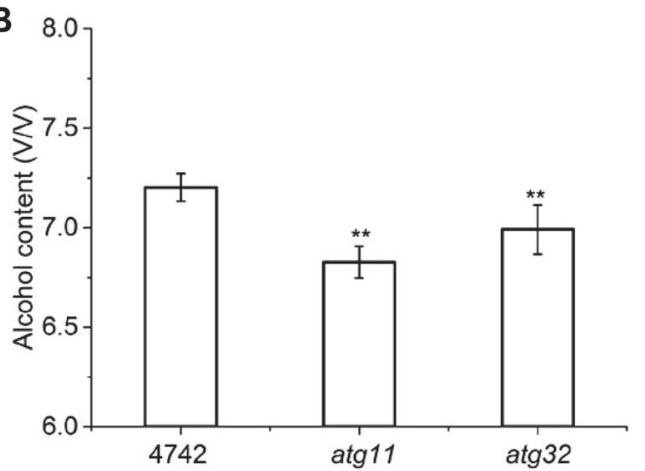

D

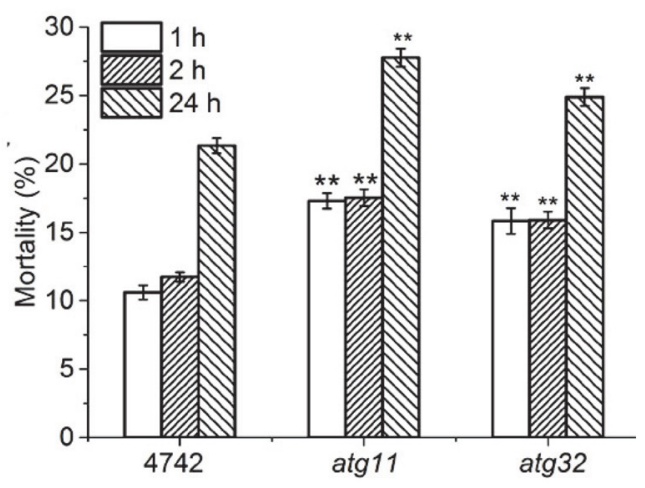

Fig. 1. Ethanol stress increased expression of the ATG11 and ATG32 genes and fermentation profiles. Normalized fold expression of the ATG11 and ATG32 were evaluated by qRT-PCR in S. cerevisiae under ethanol treated for $4 \mathrm{~h}$ (A). The gene of actin (ACT1) was used as internal reference. Ethanol yield of S.cerevisiae grown on YPD medium for $3 \mathrm{~d}$ at $30^{\circ} \mathrm{C}$ were measured by density bottle method (B). Colony morphology of yeasts grew on YPD medium with or without $10 \%$ ethanol for $3-5 \mathrm{~d}$ at $30^{\circ} \mathrm{C}(\mathbf{C})$. Mortality of cells under mid- $\log$ phase was treated with $10 \%$ ethanol for 1,2 , and $24 \mathrm{~h}$. Then the yeast were stained with PI and analyzed by flow cytometry (D). Values indicated mean \pm standard deviation $(n=3)$. Statistical significance was determined by a Student's $t$ test. Double star represented very significant between the wild type and mutants $(p<0.01)$.

membrane integrity [37-39]. As seen in Fig. 2A, ethanol stress increased fluorescence intensity in all strains. However, the relative fluorescence intensity of $\operatorname{atg} 11$, atg 32 was greater compared to the wild type. Compared to wild type, the relative fluorescence intensity of atg11, atg32 was $131.4 \%$ and $123.2 \%$ under ethanol stress, respectively (Fig. 2B). So, ethanol stress destroys membrane integrity in all strains and mitophagy is involved in the maintenance of yeast membrane integrity.

The mitochondrial inner membrane exhibits vital roles in mitochondrial function, such as with the electron
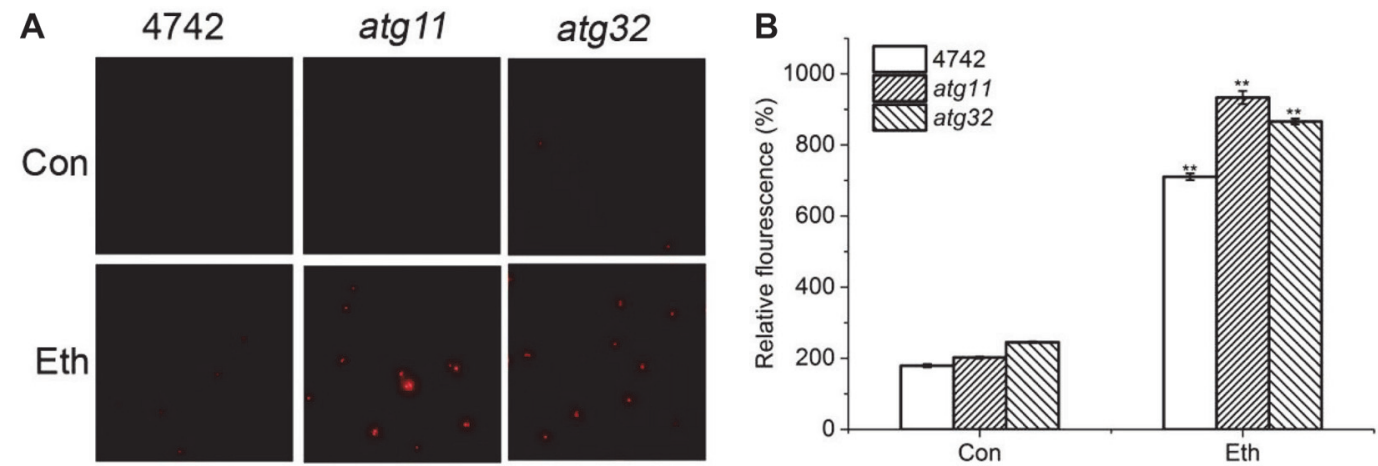

Fig. 2. Ethanol stress injures membrane integrity of yeast. Membrane integrity of WT strans, atg 11 and atg 32 mutants with $10 \%$ ethanol for $2 \mathrm{~h}$ were stained by PI, and then analyzed by fluorescence microscope $(\mathrm{A})$ and fluorescence microplate reader $(\mathbf{B})$. Values indicated mean \pm standard deviation $(n=6)$. Statistical significance was determined by a Student's $t$ test. Double star represented very significant between the wild type and mutants $(p<0.01)$. 


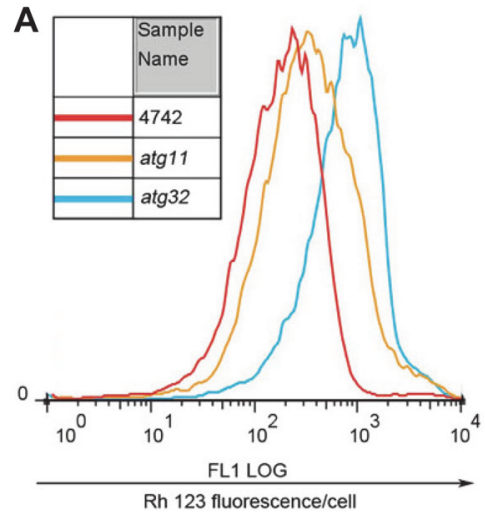

B

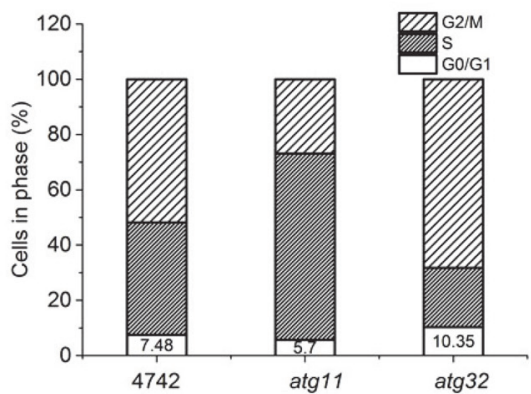

C

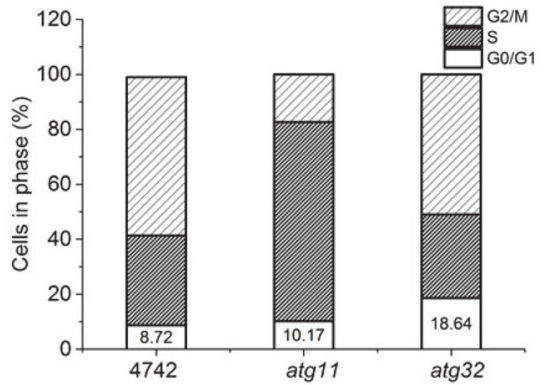

Fig. 3. Effects of mitophagy on mitochondrial membrane potential and cell cycle of S. cerevisiae under ethanol stress. Yeasts were stained with Rh123 to detect mitochondrial membrane potential. The fluorescence of S. cerevisiae with ethanol for 4 h were obtained by FACS green fluorescence histograms (FL1 log) (A). Cell cycle profile of the WT, atg11 and atg32 grown on YPD without (B) or with (C) 10\% ethanol for 4h were analyzed by flow cytometry analysis. The percentages of wild type and mutants in G1/G0, S and G2/M phases were summarized from flow cytometry analysis, respectively.

transfer chain and transmembrane transport [41]. To further explore the impact of ethanol-induced mitophagy, mitochondrial membrane potential of $S$. cerevisiae were analyzed. Compared with wild type, the mitochondrial inner membrane of the mitophagy mutants especially atg32, exhibited greater damage from ethanol stress (Fig. 3A). This indicates that mitophagy assists in maintaining the integrity of the mitochondrial inner membrane. Therefore, ethanol stress not only destroys the plasma membrane but also damages the inner membrane of mitochondrial.

It has been reported that nitrogen deficiency arrests the cell cycle in G1/G0 and that autophgy is required for G1/G0 quiescence [30, 42]. In comparison to the control fermentation, ethanol stress caused cell cycle arrest in phases G0/G1 in all strains (Figs. 3B and 3C). The cell cycle arrest of mutants was more severe compared to wild

A

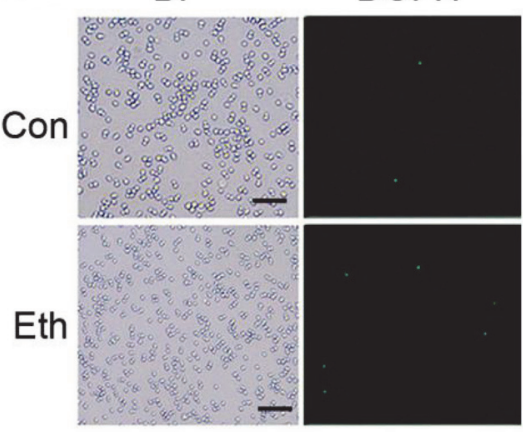

B

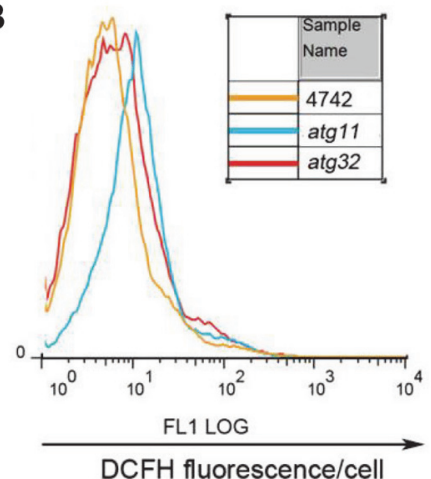

$\mathrm{BF}$

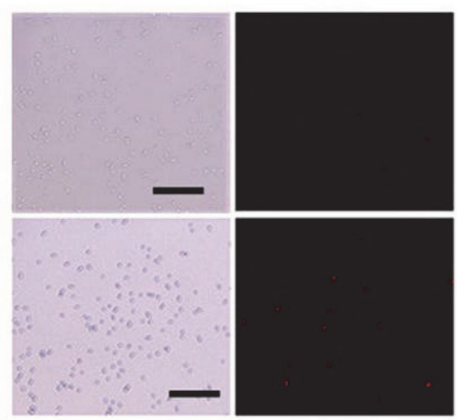

C

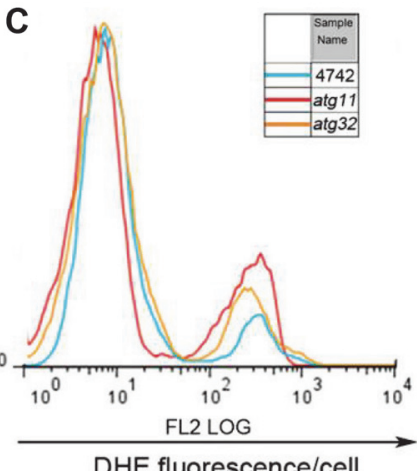

Fig. 4. Production of $\mathrm{H}_{2} \mathrm{O}_{2}$ and $\mathrm{O}_{2}^{--}$was changed in strains under ethanol stress. Production of $\mathrm{H}_{2} \mathrm{O}_{2}$ and $\mathrm{O}_{2}{ }^{--}$in wild type with ethanol for $2 \mathrm{~h}$ was stained by DCFH for $30 \mathrm{~min}$ or DHE $10 \mathrm{~min}$, respectively (A). Production of $\mathrm{H}_{2} \mathrm{O}_{2}(\mathbf{B})$ and $\mathrm{O}_{2}^{--}$(C) in WT, atg11 and atg32 with 10\% ethanol for $24 \mathrm{~h}$ was stained by DCFH or DHE and analyzed by flow cytometry. Overlay of fluorescence-activated cell sorter green or red fluorescence histograms (FL1 log) was obtained. 
type (Figs. 3B and 3C). Therefore, mitophagy contributes to ethanol tolerance in yeast by accelerating cell division, maintaining integrity of cell membrane and mitochondrial inner membrane [37, 43].

\section{Ethanol Stress Induces Production of $\mathrm{H}_{2} \mathrm{O}_{2}$ and $\mathrm{O}_{2}{ }^{-}$in S. cerevisiae}

It is established that ethanol activates oxidative stress in yeast cells during fermentation $[5,24,38]$. To evaluate whether ROS accumulation was associated with ethanol stress, ROS production was measured by fluorescence staining and flow cytometry. Production of $\mathrm{H}_{2} \mathrm{O}_{2}$ and $\mathrm{O}_{2}{ }^{-}$was induced by ethanol stress in wild type (Fig. 4A). $\mathrm{H}_{2} \mathrm{O}_{2}$ production in atg11, atg 32 was higher than that in wild type. The production of $\mathrm{H}_{2} \mathrm{O}_{2}$ in atg 32 during ethanol stress was greatest (Fig. 4B). Similarly, the $\mathrm{O}_{2}{ }^{-}$content in mutants was also higher than that in wild type (Fig. 4C). However, the difference in $\mathrm{O}_{2}$ between wild type and mutants was not as obvious as that seen with $\mathrm{H}_{2} \mathrm{O}_{2}$. Therefore, ethanol stress contributes to the accumulation of ROS and mitophagy mutants accumulate greater levels of ROS.

\section{ROS Regulates Mitophagy during Ethanol Stress}

Moderate level of ROS, as signal molecule, regulates autophagy [30]. In order to clarify the complex relationship between ROS and mitophagy under ethanol stress, reducing agents and inhibitors of antioxidase were used to alter ROS content. The common reducing agents GSH and NAC clearly reduced the production of $\mathrm{H}_{2} \mathrm{O}_{2}$ and $\mathrm{O}_{2}-$ when applied to ethanol treated yeast cells for $2 \mathrm{~h}$ (Fig. 5A). Corresponding with a decrease of ROS, GSH and NAC significantly decreased gene expression of ATG11 and ATG32 (Fig. 5B). For example, GSH decreased the expression of ATG11 and ATG32 to $91.5 \%$ and $75.8 \%$ during ethanol stress, and NAC decreased ATG11 and ATG32 expression to $38.3 \%$ and $44.1 \%$. Therefore, GSH and NAC decrease mitophagy by attenuating the production of $\mathrm{H}_{2} \mathrm{O}_{2}$ and $\mathrm{O}_{2}$.

2-ME, as the inhibitor of $\mathrm{Mn}$-SOD in mitochondria, resulted in an increase of $\mathrm{O}_{2}{ }^{-}$in mitochondria (Fig. 5C). 3AT, as the inhibitor of CAT, contributed to the rise of $\mathrm{H}_{2} \mathrm{O}_{2}$ in cytoplasm (Fig. 5C). Results indicated that 2-ME and 3-AT both increased expression of ATG11 and ATG32 genes exposed to ethanol stress (Fig. 5D). The expression of ATG11 and ATG32 increased to $207.4 \%$ and $134.1 \%$ by 2-ME. And 3-AT increased ATG11 and ATG32 expression to $153.9 \%$ and $144.7 \%$. So, 2-ME and 3-AT increase mitophagy by augmenting the production of $\mathrm{H}_{2} \mathrm{O}_{2}$ and $\mathrm{O}_{2}$.
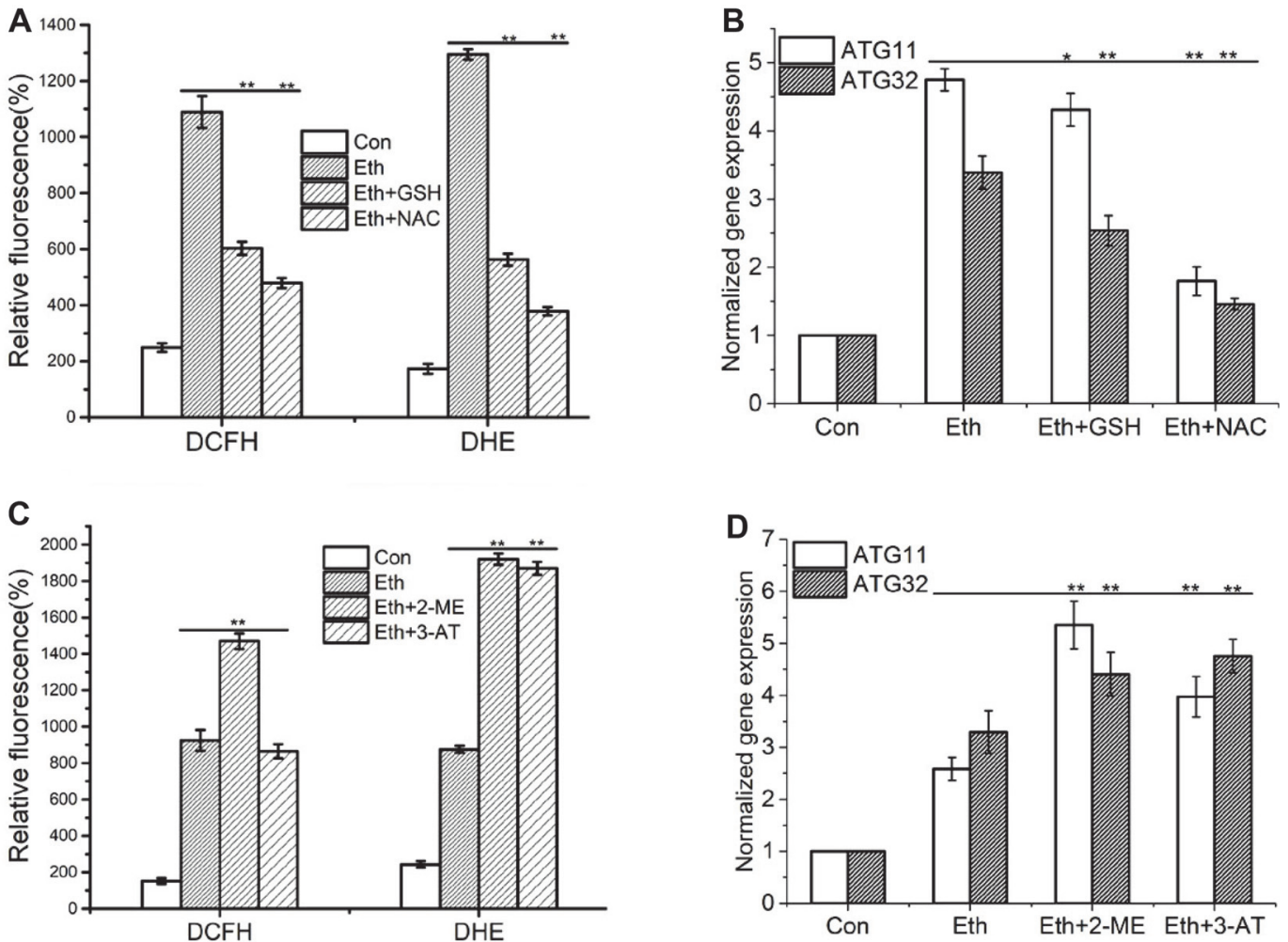

Fig. 5. Reductants and antioxidase inhibitors regulated expressions of the ATG11 and ATG32 genes and contents of $\mathrm{H}_{2} \mathrm{O}_{2}$ and $\mathrm{O}_{2}{ }^{-1}$ in S. cerevisiae cells under ethanol stress. The level of $\mathrm{H}_{2} \mathrm{O}_{2}$ and $\mathrm{O}_{2}{ }^{-}$in wild type cells treated the reductants (A) or antioxidase inhibitors (C) for $2 \mathrm{~h}$ were stained by DCFH or DHE, respectively. The relative fluorescence was summerized in A and C ( $n=6)$. The reductants further decreased expressions of ATG11 and ATG32 (B) and the antioxidase inhibitors induced expression of ATG11 and ATG32 (D). Normalized fold expression levels of the ATG11 and ATG32 genes were evaluated by qRT-PCR in S. cerevisiae with ethanol treated for $4 \mathrm{~h}$. The gene of actin, ACT1, was used as internal reference. Values indicated mean \pm standard deviation $(n=3)$. Statistical significance was determined by a Student's $t$ test. Double star or single star represented very significant $(p<0.01)$ or significant $(p<0.05)$. 
A
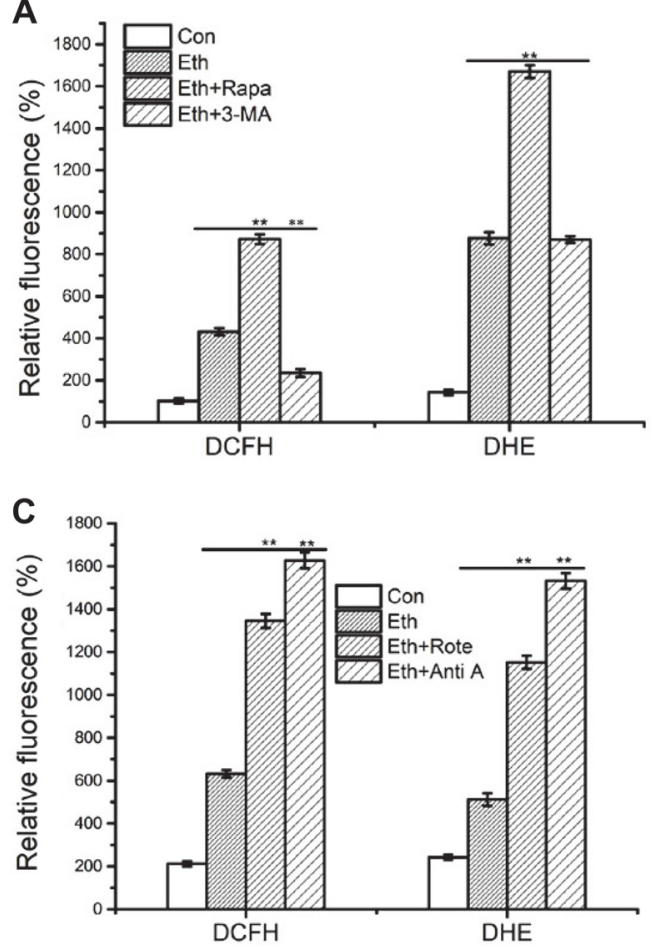

B
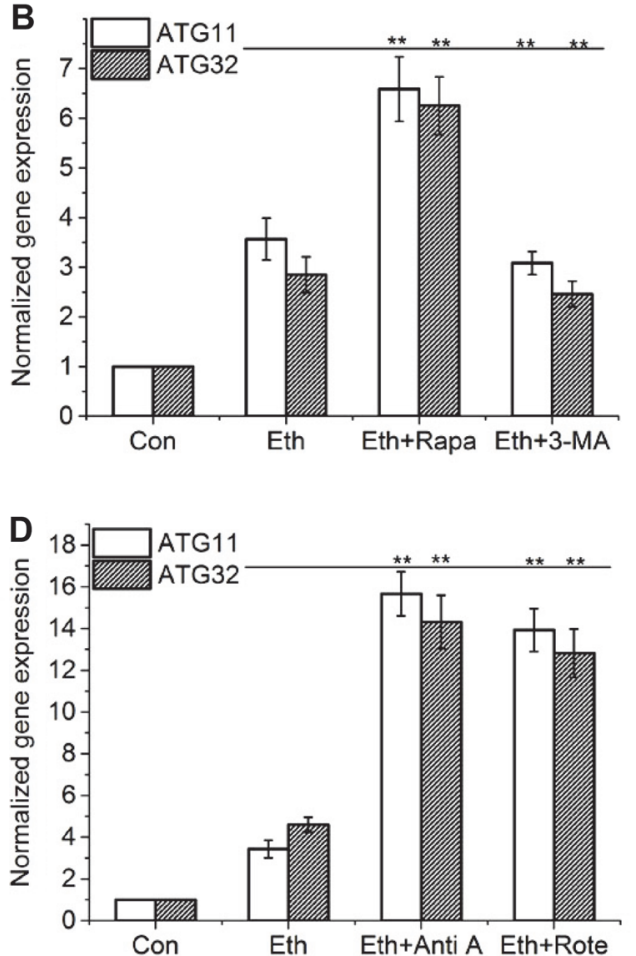

Fig. 6. The respiratory chain inhibitors or autophagy regulators regulated expression of the ATG11 and ATG32 genes and contents of $\mathrm{H}_{2} \mathrm{O}_{2}$ and $\mathrm{O}_{2}{ }^{--}$in S. cerevisiae cells under ethanol stress. Contents of $\mathrm{H}_{2} \mathrm{O}_{2}$ and $\mathrm{O}_{2}{ }^{-\cdots}$ in wild type cells treated with Rapa and 3-MA (A) or Rote and Anti A (C) for $2 \mathrm{~h}$ were stained by DCFH or DHE, respectively. The relative fluorescence was summarized in A and $\mathrm{C}(n=6)$. Rapa and 3-MA had dual functions on expression ATG11 and ATG32 (B). Rote and Anti A further induced expression of ATG11 and ATG32 genes (D). Normalized fold expression levels of the ATG11 and ATG32 gene were evaluated by qRT-PCR in S. cerevisiae under ethanol treated for 4h. The gene of actin, ACT1, was used as internal reference. Values indicated mean \pm standard deviation $(n=3)$. Statistical significance was determined by a Student's t test. Double star represented very significant $(p<0.01)$.

$\mathrm{H}_{2} \mathrm{O}_{2}$ in the yeast mitochondrial matrix plays a signaling role in ethanol tolerance through the activation of Yap1p [5]. It is well known that ROS regulates mitophagy. For example, mitophagy is positively affected by cellular oxidative stress [10]. NAC and cysteine inhibited mitophagy due to increasing cellular levels of reduced glutathione [44] and also decreased the expression of Atg32 [45]. The current results support these findings. Both GSH and NAC inhibited the expression of the ATG32 and ATG11 genes (Fig. 5B) by reducing the production of $\mathrm{H}_{2} \mathrm{O}_{2}$ and $\mathrm{O}_{2}{ }^{-}$(Fig. 5A). In contrast, the antioxidase inhibitors 2-ME and 3-AT increased expression of the ATG32 and ATG11 genes (Fig. 5D) due to increase production of $\mathrm{H}_{2} \mathrm{O}_{2}$ and $\mathrm{O}_{2}{ }^{-}$(Fig. 5C). Therefore, levels of $\mathrm{H}_{2} \mathrm{O}_{2}$ and $\mathrm{O}_{2}{ }^{-}$ are capable of regulating mitophagy in yeast exposed to ethanol stress.

Lastly, to demonstrate the ROS regulation of mitophagy in S. cerevisiae under ethanol stress, an inhibitor of autophagy 3-MA and inducer of autophagy Rapa were added to the medium under ethanol stress, respectively. Results indicated Rapa promoted the increase expression of ATG11 and ATG32 genes to $184.7 \%$ and $219.6 \%$ under ethanol treatment for $2 \mathrm{~h}$, respectively (Fig. 6B). Therefore, the inducer of autophagy is also a mitophagy activator. Similarly, Rapa promoted the production of $\mathrm{H}_{2} \mathrm{O}_{2}$ and $\mathrm{O}_{2}^{--}$under ethanol treatment for $2 \mathrm{~h}$ (Fig. 6A). In contrast, an inhibitor of autophagy 3-MA decreased the expression of ATG11 and ATG32 genes under ethanol stress (Fig. 6B). The increases of $\mathrm{H}_{2} \mathrm{O}_{2}$ and $\mathrm{O}_{2}{ }^{-}$level by ethanol were also decreased by 3-MA (Fig. 6A). In addition, Rapa increased the death rate of yeast cells whereas 3-MA decreased the rate (Fig. S3A). In summary, the inducing of mitophagy by ethanol activates yeast cell death, results in decrease of alcohol output. Therefore, regulators of autophagy also control levels of mitophagy through altering levels of ROS molecules in yeast. And mitophagy responds to varying levels of mitochondrial oxidative stress to control the quality and quantity of mitochondria [7].

The Inducing ROSs by Ethanol Are Mainly Derived from mtETC

Anti A and Rote are the inhibitors of complex III and complex I of mtETC. The result indicated that Anti A and Rote both increased the production of $\mathrm{H}_{2} \mathrm{O}_{2}$ and $\mathrm{O}_{2}{ }^{-}$(Fig. 6C) and increased the expression of ATG11 and ATG32 genes exposed to ethanol stress (Fig. 6D). Compared with ethanol stress, Anti A induced expression levels of ATG11 and ATG32 to $456.3 \%$ and $310.9 \%$, and Rote increased ATG11 and ATG32 expression to $387.8 \%$ and 278.3\%. In addition, Anti A and Rote augmented yeast cell death (Fig. S3B) and substantially lowered the alcohol 
yield (Fig. S4). Therefore, ROSs derived from mtETC regulate mitophagy and play a critical role in mediating alcohol production.

Mitophagy facilitates $\mathrm{H}_{2} \mathrm{O}_{2}$ and $\mathrm{O}_{2}$ - clearance during ethanol exposure. Similar conclusion has been reported by Kurihara et al. [13] where mitophagy decreases ROS production from mitochondria. In addition, several studies have reported that mitophagy promotes the elimination of ROS through the removal of damaged mitochondria $[9,10,13,47]$. Elevated respiration rates stimulate mitophagy degradation of the complex I equivalent and complex III of mtETC [27]. Therefore, the complex I equivalent and complex III are key mediators of ethanol stress related changes. Anti A and Rote, as inhibitors of complex III and complex I of the mtETC, were used to increased ROS. Further investigation revealed that Anti A and Rote activated mitophagy (Fig. 6D) by means of increasing the production of ROS in mitochondria (Fig. 6C). It is concluded that ROSs derived from mtETC participate in the regulation of ethanol-induced mitophagy. Similar results of Anti A induced mitophagy have been reported [46].

In conclusion, mitophagy is a complex cellular activity in the yeast. Mitochondrial oxidative stress induced by mild ethanol, as a prosurvival response during the early stages of fermentation, activates mitophagy. Mitophagy contributes to the clearance of ROS and protects yeast cells from ROS damage. These phenomena results in improved cell membrane integrity, maintenance of the mitochondrial membrane potential, decreased cell mortality, and ultimately enhanced alcohol output. Mitophagy in yeasts is regulated by ROS level in the cell during ethanol exposure. In addition, recent research indicates that an ATG32-independent pathway also contributes to the removal of mitochondria [48]. Whether Atg32 independent mitophagy induced by ethanol during fermentation requires further study.

\section{Acknowledgments}

This work was supported by projects the National Natural Science Foundation of China (31201409), Natural Science Project of Henan Science and Technology Department (162300410175). The work also was funded by National Key Laboratory of Wheat and Maize Crop Science in Henan.

\section{Conflict of Interest}

The authors have no financial conflicts of interest to declare.

\section{References}

1. Muller FL, Liu Y, Van Remmen H. 2004. Complex III releases superoxide to both sides of the inner mitochondrial membrane. J. Biol. Chem. 279: 49064-49073.

2. Fedoseeva IV, Pyatrikas DV, Stepanov AV, Fedyaeva AV, Varakina NN, Rusaleva TM, et al. 2017. The role of flavin-containing enzymes in mitochondrial membrane hyperpolarization and ROS production in respiring Saccharomyces cerevisiae cells under heatshock conditions. Sci. Rep. 7: 2586 .

3. Miller A. 2012. Superoxide dismutases: ancient enzymes and new insights. FEBS. Lett. 586: 585-595.

4. Kwon YY, Choi KM, Cho CY, Lee CK. 2015. Mitochondrial efficiency-dependent viability of Saccharomyces cerevisiae mutants carrying individual electron transport chain component deletions. Mol. Cells 38: 1054-1063.

5. Zyrina AN, Smirnova EA, Markova OV, Severin FF, Knorre DA. 2017. Mitochondrial superoxide dismutase and Yap1p act as a signaling module contributing to ethanol tolerance of the yeast Saccharomyces cerevisiae. Appl. Environ. Microbiol. 83 Pii: e02759-16.

6. Magrì A, Di Rosa MC, Tomasello MF, Guarino F, Reina S, Messina A, et al. 2016. Overexpression of human SOD1 in VDAC1-less yeast restores mitochondrial functionality modulating beta-barrel outer membrane protein genes. Biochim. Biophys. Acta 1857: 789798.

7. Knuppertz L, Warnsmann V, Hamann A, Grimm C, Osiewacz HD. 2017. Stress-dependent opposing roles for mitophagy in aging of the ascomycete Podospora anserina. Autophagy 13: 1037-1052

8. Rattanawong K, Kerdsomboon K, Auesukaree C. 2015. Cu/Zn-superoxide dismutase and glutathione are involved in response to oxidative stress induced by protein denaturing effect of alachlorin Saccharomy cescerevisiae. Free. Radical. Biol. Med. 89: 963-971.

9. Fukuda T, Kanki T. 2018. Mechanisms and physiological roles of mitophagy in yeast. Mol. Cells 41: 35-44.

10. Kanki T, Furukawa K, Yamashita S. 2015. Mitophagy in yeast: molecular mechanisms and physiological role. Biochim. Biophys. Acta 1853: 2756-2765.

11. Fan P, Xie X, Chen CH, Peng X, Zhang P, Yang C, et al. 2019. Molecular regulation mechanisms and interactions between reactive oxygen species and mitophagy. DNA Cell Biol. 38: 10-22.

12. Müller M, Lu K, Reichert AS. 2015. Mitophagy and mitochondrial dynamics in Saccharomyces cerevisiae. Biochim. Biophys. Acta 1853: 2766-2774.

13. Kurihara Y, Kanki T, Aoki Y, Hirota Y, Saigusa T, Uchiumi T, et al. 2012. Mitophagy plays an essential role in reducing mitochondrial production of reactive oxygen species and mutation of mitochondrial DNA by maintaining mitochondrial quantity and quality in yeast. J. Biol. Chem. 287:3265-3272.

14. Tsukada M, Ohsumi Y. 1993. Isolation and characterization of autophagydefective mutants of Saccharomyces cerevisiae. FEBS. Lett. 333: $169-174$

15. Yoo SM, Jung YK. 2018. A molecular approach to mitophagy and mitochondrial dynamics. Mol. Cells 41: 18-26.

16. Xia X, Katzenell S, Reinhart EF, Bauer KM, Pellegrini M, Ragusa MJ. 2018. A pseudo-receiver domain in Atg32 is required for mitophagy. Autohhagy 14: 1620-1628.

17. Aihara M, Jin X, Kurihara Y, Yoshida Y, Matsushima Y, Oku M, et al. 2014. Tor and the Sin3-Rpd3 complex regulate expression of the mitophagy receptor protein Atg32 in yeast. J. Cell Sci. 127: 3184-3196.

18. Furukawa K, Fukuda T, Yamashita S, Saigusa T, Kurihara Y, Yoshida Y, et al. 2018. The PP2A-like protein phosphatase Ppg1 and the Far complex cooperatively counteract CK2-mediated phosphorylation of Atg32 to inhibit mitophagy. Cell Rep. 23: $3579-3590$.

19. Shan YT, Gu ZW, Kong Z, Zhou Y, Li B., Yan MF, et al. 2019. Progress on function of ATG11. Prog. Biochem. Biophys. 46: 796-803.

20. Zientara-Rytter K, Ozeki K, Nazarko TY, Subramani S. 2018. Pex3 and Atg37 compete to regulate the interaction between the pexophagy receptor, Atg30, and the Hrr25 kinase. Autophagy 14: 368-384.

21. Chen S, Cui Y, Parashar S, Novick PJ, Ferro-Novick S. 2018. ER-phagy requires Lnp1, a protein that stabilizes rearrangements of the ER network. Proc. Natl. Acad. Sci. USA 115: E6237-E6244. 
22. Eapena VV, Waterman DP, Bernard A, Schiffmann N, Sayasd E, Kamber R, et al. 2017. A pathway of targeted autophagy is induced by DNA damage in budding yeas. Proc. Natl. Acad. Sci. USA 116: E1158-E1167.

23. Waliullah TM, Yeasmin AMST, Kaneko A. 2017. Rim15 and Sch9 kinases are involved in induction of autophagic degradation of ribosomes in budding yeast. Biosci. Biotech. Biochem. 81:307-310.

24. Takagi H, Taguchi J, Kaino T. 2016. Proline accumulation protects Saccharomyces cerevisiae cells in stationary phase from ethanol stress by reducing reactive oxygen species levels. Yeast 33: 355-363.

25. Noctor G, Foyer, CH. 1998. Ascorbate and glutathione: keeping active oxygen under control. Annu. Rev. Plant Physiol. Plant Mol. Biol. 49: 249-279.

26. Tesnière C, Brice C, Blondin B. 2015. Responses of Saccharomyces cerevisiae to nitrogen starvation in wine alcoholic fermentation. Appl. Microbiol. Biotechnol. 99: 7025-7034.

27. Timón-Gómez A, Sanfeliu-Redondo D, Pascual-Ahuir A, Proft M. 2018. Regulation of the stress-activated degradation of mitochondrial respiratory complexes in yeast. Front. Microbiol. 9: 106.

28. Shiroma S, Jayakody LN, Horie K, Okamoto K, Kitagakia H. 2014. Enhancement of ethanol fermentation in Saccharomyces cerevisiae sake yeast by disrupting mitophagy function. Appl. Environ. Microbiol. 80: 1002-1012.

29. Jing HJ, Liu HH, Zhang L, Gao J, Song HR, Tan XR. 2018. Ethanol induces autophagy regulated by mitochondrial ROS in Saccharomyces cerevisiae. J. Microbiol. Biotechnol. 28: 1982-1991.

30. Mendes-Ferreira A, Sampaio-Marques B, Barbosa C, Rodrigues F, Costa V, Mendes-Faia, A, et al. 2010. Accumulation of nonsuperoxide anion reactive oxygen species mediates nitrogen-limited alcoholic fermentation by Saccharomyces cerevisiae. Appl. Environ. Microbiol. 76: 7918-7824.

31. Almeida B, Sampaio-Marques B, Carvalho J, Silva MT, Leao C, et al. 2007. An atypical active cell death process underlies the fungicidal activity of ciclopirox olamine against the yeast Saccharomyces cerevisiae. FEMS Yeast Res. 7: 404-412.

32. Kainz K, Tadic J, Zimmermann A, Pendl T, Carmona-Gutierrez D, Ruckenstuhl C, Eisenberg T, et al. 2017. Methods to assess autophagy and chronological aging in yeast. Methods Enzymol. 588: 367-394.

33. Ludovico P, Rodrigues F, Almeida A, Silva MT, Barrientos A, Corte-Real M. 2002. Cytochrome c release and mitochondria involvement in programmed cell death induced by acetic acid in Saccharomyces cerevisiae. Mol. Biol. Cell. 13:2598-2606.

34. Ludovico P, Sansonetty F, Corte-Real M. 2001. Assessment of mitochondrial membrane potential in yeast cell populations by flow cytometry. Microbiology 147: 3335-3343.

35. Schmittgen TD, Livak KJ. 2008. Analyzing real-time PCR data by the comparative CT method. Nat. Protoc. 3: 1101-1108.

36. Wagner-Vogel G, Lämmer F, Kämper J, Basse CW. 2015. Uniparental mitochondrial DNA inheritance is not affected in Ustilago maydis $\triangle$ atg11 mutants blocked in mitophagy. BMC Microbiol. 15: 23.

37. Ma M, Liu ZL. 2010. Mechanisms of ethanol tolerance in Saccharomyces cerevisiae. Appl. Microbiol. Biotechnol. 87: 829-845.

38. Charoenbhakdi S, Dokpiku T, Burphan T, Techo T, Auesukaree C. 2016. Vacuolar H ${ }^{+}$-ATPase protects Saccharomyces cerevisiae cells against ethanol-induced oxidative and cell wall stresses. Appl. Environ. Microbiol. 82: 3121-3130.

39. Dog?an A, Demi?rci? S, Ayteki?n AÖ, S?ahi?n F. 2014. Improvements of tolerance to stress conditions by genetic engineering in Saccharomyces cerevisiae during ethanol production. Appl. Biochem. Biotechnol. 174: 28-42.

40. Lin Y, Tanaka S. 2006. Ethanol fermentation from biomass resources: current state and prospects. Appl. Microbiol. Biotechnol. 69: 627-642.

41. Zorova LD, Popkov VA, Plotnikov EY, Silachev DN, Pevzner IB, Jankauskas SS, et al. 2018. Mitochondrial membrane potential. Analytic. Biochem. 552: 50-59.

42. An Z, Tassa A, Thomas C, Zhong R, Xiao G, Fotedar R, et al. 2014. Autophagy is required for G1/G0 quiescence in response to nitrogen starvation in Saccharomyces cerevisiae. Autophagy 10: 1702-1711.

43. Kim S, Kim J, Song JH, Jung YH, Choi IS, Choi W, et al. 2016. Elucidation of ethanol tolerance mechanisms in saccharomyces cerevisiae by global metabolite profiling. Biotechnol. J. 11: 1221-1229.

44. Deffieu M, Bhatia-Kissova I, Salin B, Galinier A, Manon S, Camougrand N. 2009. Glutathione participates in the regulation of mitophagy in yeast. J. Biol. Chem 284: 14828-14837.

45. Okamoto K, Kondo-Okamoto N, Ohsumi Y. 2009. Mitochondria-anchored receptor Atg32 mediates degradation of mitochondria via selective autophagy. Dev. Cell. 17: 87-97.

46. Lazarou M, Sliter DA, Kane LA, SarrafSA, Wang C, Burman JL, et al. 2015. The ubiquitin kinase PINK1 recruits autophagy receptors to induce mitophagy. Nature 524: 309-314

47. Bin-Umera MA, McLaughlina JE, Butterlya MS, McCormickb S, Tumer NE. 2014. Elimination of damaged mitochondria through mitophagy reduces mitochondrial oxidative stress and increases tolerance to trichothecenes. Proc. Natl. Acad. Sci. USA 111: 1179811803

48. Yao Z, Klionsky DJ. 2016. An unconventional pathway for mitochondrial protein egradation. Autophagy 12: 1971-1972. 